\title{
When Colours split from Objects: The Disconnection of Colour Perception from Colour
}

\section{Language and Colour Knowledge}

Katarzyna Siuda-Krzywicka ${ }^{*}$, Christoph Witzel ${ }^{2}$, Myriam Taga $^{3}$, Marine Delanoe ${ }^{4}$, Laurent Cohen $^{5,6}, \&$ Paolo Bartolomeo ${ }^{7}$

1. Inserm U 1127, CNRS UMR 7225, Sorbonne Université, Institut du Cerveau et de la Moelle épinière, ICM, Hôpital de la Pitié-Salpêtrière, 47 Boulevard de l'Hopital, 75013 Paris, France, siuda.krzywicka@gmail.com

2. Justus-Liebig Universität Gießen, FB 06 Psychologie und Sportwissenschaft, OttoBehaghel-Str. 10F, 35394 Gießen, cwitzel@daad-alumni.de

3. University of East London, University Way, Royal Docks, London E16 2RD, UK, myriam.taga@gmail.com

4. Inserm U 1127, CNRS UMR 7225, Sorbonne Université, Institut du Cerveau et de la Moelle épinière, ICM, Hôpital de la Pitié-Salpêtrière, 47 Boulevard de l'Hopital, 75013 Paris, France, marinedelanoe@ hotmail.com

5. Inserm U 1127, CNRS UMR 7225, Sorbonne Université, Institut du Cerveau et de la Moelle épinière, ICM, Hôpital de la Pitié-Salpêtrière, 47 Boulevard de l'Hopital, 75013 Paris, France, laurentcohen2@gmail.com

6. Hôpital de la Pitié-Salpêtrière, 47 Boulevard de l'Hopital, 75013 Paris, France Paris, France

7. Inserm U 1127, CNRS UMR 7225, Sorbonne Université, Institut du Cerveau et de la Moelle épinière, ICM, Hôpital de la Pitié-Salpêtrière, 47 Boulevard de l'Hopital, 75013 Paris, France, paolo.bartolomeo@gmail.com *corresponding author 


\begin{abstract}
We investigated object-colour knowledge in RDS, a patient with impaired colour naming after a left occipito-temporal stroke. RDS's colour perception, object naming and verbal colour-knowledge (the ability to verbally say the typical colour of an object) were relatively spared. RDS was also able to state if an object was appropriately coloured or not. However, he could neither match colour names to coloured objects, nor match colour patches to greyscale objects. Thus, RDS's colour-naming deficit was associated with an impaired ability to conceptually relate visually presented object shapes and colours. These results suggest that objects in their typical colour are processed holistically in the visual modality, and that abilities important for colour naming may also be involved in abstracting colours from visual objects. We discuss these findings in the context of developmental psychology and linguistic anthropology, and propose a model of neuro-functional organization of object-colour knowledge.
\end{abstract}

\title{
Keywords
}

colour knowledge; colour naming; language; concepts; visuo-verbal disconnection; inferior temporal cortex; ventral cortical visual stream 


\section{Introduction}

Colour cognition has often served as a probe to investigate the extent to which language can interact with perception and other cognitive systems. In particular, it has been debated weather colour naming and categorisation can influence colour perception (reviewed in: Davidoff, 2001; Regier \& Kay, 2009; Siuda-Krzywicka, Boros, Bartolomeo, \& Witzel, 2019; Witzel, 2018; Witzel \& Gegenfurtner, 2018). Here, we investigated how language interacts with knowledge about the colours of objects.

Some objects, such as animals, plants and fruits, are typically associated with a specific colour. Such objects are dubbed colour-diagnostic and the associated colours is their memory colour (Witzel \& Gegenfurtner, 2014). Neuropsychological evidence shows that knowledge of memory colours can be stored in verbal and visual formats (reviewed in: Tanaka, Weiskopf, \& Williams, 2001). Verbal colour knowledge refers to purely verbal associations between colour names and object names (e.g. strawberry - red, or banana yellow). Visual colour knowledge refers to visual representations of memory colours. There are two competing views on the organization of visual colour knowledge. According to the edge-based theories of visual recognition (Biederman \& Ju, 1988), visual colour knowledge could be organized as a system of associations between non-coloured representations of object shapes (edges) and the representations of colours. Alternatively, the Shape + Surface model claims that, at least for colour-diagnostic objects, colour is an integral part of object representation, and that visual colour knowledge stores colour and shape of an object in an integrated way (Figure 1; see Tanaka et al., 2001 for review).

[Figure 1 somewhere here]

Visual colour knowledge is assumed to be acquired through frequent visual experience with colour diagnostic objects (Bartleson, 1960; Witzel \& Gegenfurtner, 2014; Witzel \& Hansen, 2015). However, evidence from young children and neurological patients suggests 
that language could play a role in its acquisition and retrieval. Young children (2-5 years old) and aphasic patients have difficulties when differentiating between congruently and incongruently coloured line-drawings of colour diagnostic objects and when colouring line drawings of colour-diagnostic objects (Basso, Capitani, Luzzatti, Spinnler, \& Zanobio, 1985; Basso, Faglioni, \& Spinnler, 1976; Cohen \& Kelter, 1979; Davidoff \& Mitchell, 1993; De Renzi \& Spinnler, 1967; Gleason, Fiske, \& Chan, 2004). The connection between objects and their typical colours could thus be at least partially mediated by language.

Here, we assessed the two rival models of visual colour knowledge, as well as the role of language in its formation and maintenance, in a stroke patient, RDS. As a consequence of a left occipito-temporal lesion, RDS presented a right homonymous hemianopia and a selective inability to name visually presented colours and orthographic material. Neuropsychological assessment showed that RDS's naming impairment was colour-specific, with spared naming of objects and faces. RDS's lesion did not cause dramatic impairments in colour-vision, neither did it affect his colour lexicon. RDS was able to freely recall colour names in a colourfluency test and associate colour names with object names, showing spared verbal colour knowledge. Thus, in RDS perceived colours were disconnected from colour names (Geschwind \& Fusillo, 1966).

We investigated how RDS's visuo-verbal disconnection interacts with his colour knowledge in three experiments. In experiment 1, we assessed RDS's visual recall of memory colours under two conditions: when colours were presented on objects, or when colours were presented as colour patches next to achromatic object shapes. If visual colour knowledge is stored as associations between separate representations of object shapes and colours, RDS's performance should be comparable on both conditions. A dissociation between the two conditions would suggest the functional segregation between processing of coloured objects and linking object's shapes and colours. In the second experiment we assessed RDS's general 
abilities to simultaneously process multiple visual stimuli, by asking him to judge the congruency of simultaneously presented object shapes and textures, and different object parts.In the third experiment (colour-name matching), we assessed whether or not RDS can use his spared object identification and verbal colour knowledge to facilitate colour naming. We asked RDS to match colour names to colour patches, typically coloured objects and typical colours of objects presented in grey scale.

Our results show that RDS had spared knowledge of typically coloured objects, which he was not able to break down to separate features of colour and shape. Our findings support the Shape+Surface model of the object-colour knowledge organisation; and imply that language contributes to breaking down object representation to separate features of colour and shape.

\section{Case Report}

RDS, a right-handed 54-year-old man, had an ischemic stroke in the territory of the left posterior cerebral artery in February 2014. Before the stroke, he had worked with colours daily as a manager and car paint expert in an insurance company. After the stroke, RDS presented with right homonymous hemianopia, alexia without agraphia and difficulties in naming visually presented colours. When tested, he was well oriented in time and space, fully cooperative, and had preserved general intellectual abilities. The testing took place between 5 and 55 months post-stroke.

\section{Lesion Location}

RDS's lesion was situated in the left mesial occipito-temporal cortex. It encompassed the calcarine sulcus, the lingual, fusiform and parahippocampal gyri in the left hemisphere, as well as the splenium of corpus callosum (Figure 2). The lesion damaged the left primary and secondary visual cortices, causing complete hemianopia without macular sparing. 
[Figure 2 somewhere here]

\section{General Intellectual Abilities}

RDS performed the Wechsler Adult Intelligence Scale, fourth edition (WAIS-IV) 46 months post-stroke. He exhibited normal performance on the subscales measuring verbal comprehension (score 94, 88-101 95\% CI, 34th percentile), and working memory (score 91, 84-99 95\%CI, 27th percentile). His perceptual reasoning skills were within the low average (score: 86, 80-93 95\%CI, 18th percentile). His performance was impaired on the Visual Puzzles (7/26, standard score 5), and on the Block Design tasks (32/66, standard score 7). This suggests some difficulties in assessing the relationships between parts, simultaneously processing multiple visual stimuli and visualizing spatial conformations. RDS's relatively high score in Matrix Reasoning task (20/26, standard score 11) indicates normal non-verbal concept formation. Finally, RDS was severely impaired in the processing speed subscale (score 61, 57-71 95\%CI, 0.5th percentile), likely because of his reading disorder.

\section{Colour Perception and Naming}

Details on RDS's colour perception and naming can be found in Siuda-Krzywicka et al (submitted). Briefly, RDS showed signs of a yellow-blue dyschromatopsia, likely associated with his type II diabetes (O’Neill-Biba, Sivaprasad, Rodriguez-Carmona, Wolf, \& Barbur, 2010). His red-green colour perception was normal. Fifty-two months post-stroke, RDS was able to name only $34 \%$ of visually presented, chromatic colours.

\section{Colour-name Fluency}

RDS was asked to freely recall as many colour names as possible in one minute. He provided 13 different colour names in the following order: white, black, blue, red, green, yellow, purple, indigo, violet, grey, anthracite, pink, orange. The published controls' mean 
score on such colour fluency task is $10.41 \pm 2.15$ (De Vreese, 1991). This result indicates that RDS had preserved to colour lexicon.

\section{Verbal Colour Knowledge}

To assess RDS's verbal colour-knowledge, we asked him to say the appropriate colour of 20 objects named by the examiner. We used the list of 20 objects published by Bartolomeo et al. (1997, see Bourlon et al. 2009, for normative data). RDS responded correctly on 16 out of 20 trials. For instance, he was able to say that a cotton wool is typically white, a flamingo is pink, or that a tree's trunk is brown. His errors concerned the following objects: tarantula (he couldn't recall what the animal looked like), pumpkin ("brown"), strawberry ("pink") and dolphin ("white", the correct response being grey). Although not dramatically impaired, RDS's overall performance was lower than the published norm (19.13 \pm 0.8 ; (Bourlon et al., 2009), $\mathrm{t}(24)=-3.83, \mathrm{p}<0.001, \mathrm{z}=-3.91)$.

\section{Object Naming}

Six months post-stroke, RDS presented signs of optic aphasia, a naming impairment specific to visually presented objects (Rodrigues et al., 2008). For example, when shown a black-and-white image of a sled, he said: "Something to protect yourself, something you give to the kids when they go to the pool, a bathrobe, to protect themselves from the snow, the cold, to slide on the snow, to go sledding... a sled". At this time, he correctly named 16 out of $24(66 \%)$ images of animals, fruits and vegetables, and man-made objects from the Snodgrass and Vanderwart pictorial set (Snodgrass \& Vanderwart, 1980). We tested his object naming again 38 months post-stroke. RDS was asked to name a set of 80 black-and-white images and grey-scale photographs of objects, including natural and man-made items. Note that the same set of object images was used in experiments 1 and 3. RDS named correctly all the objects, thus showing full recovery from the optic aphasia he had presented in the subacute stage. 


\section{Face Naming}

We examined RDS's face identification 11 months post-stroke. We asked him to identify a set of 20 coloured photographs of famous people and compared his naming accuracy to the normative data collected by Bourlon et al. (2009). RDS correctly identified all the presented faces (healthy subjects ages 31-60 identified 19 \pm 1 images, see Bourlon et al, 2009).

\section{General experimental methods}

\section{Participants}

RDS and several groups of healthy controls participated in the different measurements reported below. In total, these groups included sixteen males aged 49-59 years (mean 53.41 \pm 3.73 ), working as office workers, like RDS used to. All controls were right-handed according to the Edinburgh Inventory (Oldfield, 1971), had normal or corrected-to-normal vision, and showed normal red-green colour vision with the Ishihara Colour plates test (Ishihara, 1974). The present research was promoted by the Inserm (CPP C13-41) and approved by the Ile-de-France I ethical committee. Before participating in this research, all participants signed an informed consent form. The form was read to RDS because of his reading disorder.

\section{Materials}

The computerized tests were designed in Eprime 2 (Psychology Software Tools, Inc.) and OpenSesame (Mathôt, Schreij, \& Theeuwes, 2012) software. In all the computerized tests, the trial duration was not limited, and a subsequent trial appeared after key press. Unless stated otherwise, the stimuli were displayed on a CRT monitor, driven by a NVIDIA Ge Force GT640 graphics card with a spatial resolution of $1024 \times 758$ pixels, a refresh rate of $60 \mathrm{~Hz}$, and a colour resolution of 8 bits per channel. The viewing distance was $60 \mathrm{~cm}$. In the experiments 
assessing colour cognition colour rendering was calibrated, and gamma corrected. The CIE 1931 chromaticity coordinates and luminance for the monitor primaries were $\mathrm{R}=(0.614$, $0.356,27.3), \mathrm{G}=(0.286,0.60060 .1)$, and $\mathrm{B}=(0.146,0.070,9.4)$.

\section{Quantification and statistical analysis}

Experiments 1-3 consisted of yes/no tasks, involving congruent (signal) trials for which the correct response was "yes", and incongruent (noise) trials for which the correct response was “no". We quantified separately participants' accuracy (\% of correct responses) on congruent trials and on incongruent trails. Additionally, we applied Signal Detection Theory to analyse participants' performance in terms of sensitivity (d', describing subjects' ability to distinguish between signal and noise) and bias (c, reflecting subjects' general tendency to respond yes or no). High c values indicate a conservative bias, i.e. overall tendency to respond "no", while low c values indicate a liberal bias, with a tendency to respond "yes” (Stanislaw \& Todorov, 1999).

We performed case-control comparisons with the Crawford modified t-test (Crawford \& Howell, 1998). This test calculates the probability that the case comes from the distribution of a control sample. To test for a dissociation between two tasks or conditions, we used the Revised Standardized Difference Test (RSDT, Crawford \& Garthwaite, 2005). RSDT is a modification of a paired t-test that compares the difference between case scores on two tasks with the analogous difference in the control sample.

\section{Experiment 1: Colour congruency judgment}

\section{Methods}

\section{Participants}

RDS and 11 age-, gender- and education-matched healthy controls took part in the study. 


\section{Design}

Participants were asked to decide whether a visually presented colour was congruent with the memory colour of a visually presented objects shape. There were two experimental conditions. In the colour-on-object condition, RDS was presented with congruently and incongruently coloured images of objects. In the colour-split condition, the same colourdiagnostic objects were presented in grey scale, together with a separate colour patch containing either a congruent or an incongruent colour (see Figure 3).

\section{Materials}

We used a selection of 80 pictures of objects taken from the image sets used in NaorRaz, Tarr, \& Kersten, 2003; Rossion \& Pourtois, 2004; Witzel, Valkova, Hansen, \& Gegenfurtner, 2011. The objects' typical colours comprised 4 categories: brown, green, yellow and red. At the time of the test, RDS identified correctly all of the visually presented objects (see Case Description: Object naming). To obtain grey-scale images, we represented the colours of the images in the CIELUV space (using monitor white as white-point) and set chromatic coordinates $\left(\mathrm{u}^{*}, \mathrm{v}^{*}\right)$ to zero. For the atypically coloured images, we rotated the chromatic distributions of the colour-diagnostic features of an object (e.g. the red fruit but not the green tail of a strawberry) to the opponent areas in the CIELUV colour space to ensure that their colour could not be considered typical for a given object.

\section{Procedure}

Each trial started with a 500-ms fixation point. Then, in the colour-on-object condition, an image was presented at the monitor centre. In the colour-split condition, a greyscale object and a colour patch were presented, one under another, on the vertical meridian of the screen. Subjects responded with their right hand as to whether the object was typically coloured by pressing upper arrow for yes and lower arrow for no. Each object was presented 
in the colour-on-object and the colour-split condition, in a congruent and incongruent colour, resulting in 320 trials and a 50\% chance level. The two conditions were interleaved.

\section{Results}

Figure 3 summarizes the results. In the colour-on-object condition, on both congruent and incongruent trials, RDS's performance was above 90\% correct. Still, he was impaired as compared with controls' (congruent trials accuracy, 91\% for RDS vs. $98 \pm 2 \%$ for controls, $\mathrm{t}(10)=-3.73, \mathrm{p}=0.002, \mathrm{z}=-3.9$; incongruent trials accuracy, 92\% vs. $97 \pm 2 \%, \mathrm{t}(10)=-2.47$, $\mathrm{p}=0.016, \mathrm{z}=-2.58)$. RDS did not show dissociation in performance between incongruent and congruent trials: the difference between RDS's scores in the two conditions was not significantly larger than the analogous difference in the control group (RSDT $\mathrm{p}=0.341$ ). STD analysis revealed that RDS's sensitivity was impaired (d'=2.72 vs $4.07 \pm 0.5 \%, t(10)=-2.41$, $\mathrm{p}=0.018)$; and his response bias did not differ significantly from controls' $(\mathrm{t}(10)=0.21$, $\mathrm{p}=0.418)$.

In contrast, in the colour-split condition RDS was much less accurate on the congruent $(31 \%$ vs. $86 \pm 8 \%$ correct, $\mathrm{t}(10)=-6.73, \mathrm{p}<0.001, \mathrm{z}=-7.02)$ than on the incongruent trials (where his performance did not differ significantly from controls', $\mathrm{p}=0.207$ ), showing a dissociation (RSDT $\mathrm{p}=0.004)$. His sensitivity was impaired $\left(\mathrm{d}^{\prime}=0.92 \mathrm{vs}, 2.7 \pm 0.35, \mathrm{t}(10)=-5.02, \mathrm{p}<0.001\right)$, and he presented a conservative bias ( $c=0.96$ vs $c=0.21 \pm 0.19, t(10)=3.67, p=0.002, z=3.84)$.

[Figure 3 somewhere here]

\section{Discussion}

The results of experiment 1 show that RDS had a relatively spared ability to distinguish between congruently and incongruently coloured images of objects. However, he 
was severely impaired in judging whether colour patches depicted a memory colour of a simultaneously presented grey-scale object.

To judge if a given colour is typical for object shape, one can possibly use two strategies. According to the first strategy, consistent with edge-based theories of visual recognition (Figure 1A), a shape representation is first activated, then it is associated with a memory colour, and finally compared to the visual input (see Davidoff \& Mitchell, 1993, p 134, for discussion). In this case, we would not expect a difference in performance in the colour-on-object and colour-split conditions, because the same mental operation (matching a colour to an achromatic shape) would underlie both conditions. According to the alternative strategy, the object's image could be directly compared to a coloured template, a joint representation object and colour, in agreement with the Shape+Surface models (Figure 1B; Tanaka et al., 2001). If so, resolving the colour-on-object condition would be easier because the task would come down to comparing a mental template to a perceptual object. The coloursplit condition would be more difficult because it would require two comparisons; one between the template and the achromatic object; and second between the template and the colour patch. Our results are consistent with the second scenario, and thus support the Shape+Surface model of visual cognition.

An alternative explanation of our results could be that the patches we created did not reliably reflect the typical colour of the grey scale objects. This however is unlikely because control subjects performed at ceiling on this task. RDS could also be impaired in the coloursplit condition because of a colour-unrelated difficulty in simultaneously assessing multiple visual stimuli, such as in simultagnosia. Such impairment would also be consistent with his results on the WAIS-IV scale (see Case Report). We ran Experiment 2 to control for this possibility. 


\section{Experiment 2: Property verification}

\section{Methods}

\section{Participants}

RDS and 5 demographically-matched control subjects took part in the study. The 5 control subjects were different from those who participated in experiment 1.

\section{Design}

Participants had to judge the congruency between a visually presented object form and a texture patch (texture verification condition), or between visually presented object parts (shape verification condition). We initially aimed at testing if colour information would modulate RDS's performance in the texture-verification condition, so for each object form we created coloured and grey-scale texture patches. The results showed that colour did not significantly affect RDS's performance (see Supplementary Materials), thus we combined coloured and grey-scale textures and analysed them as a single texture-verification condition.

\section{Materials}

All images used in this experiment were downloaded from Wikimedia Commons (https://commons.wikimedia.org). In the texture-verification condition, we used a set of 21 photographs depicting animals, fruits and vegetables, and manmade objects. To obtain images including only object shape information without any surface information, for each photograph we created an object mask. For each object, we downloaded an image depicting a corresponding texture patch (e.g. bear-fur, snake-snake skin etc.). In the shape-verification condition, we used 40 photographs of animals, fruits and vegetables and manmade objects and turned them to grey-scale with MATLAB rgb2grey function. Subsequently, the object parts images were cut out by hand. 


\section{Procedure}

On each trial, two images were presented. In the texture verification condition, the image of object form and the texture patch were aligned on the central vertical meridian of the screen (like in the colour-split condition of Experiment 1). In the shape-verification condition, most of the stimuli were created by cutting the images into two parts on the vertical axis. To prevent RDS from resolving this task by making a judgment based on the perceptual Gestalt, we aligned the object parts horizontally (see Figure 4). To facilitate RDS's performance in view of his right homonymous hemianopia, in this condition the images were presented on the left side of the screen. Participants responded with their right hand as to whether the texture patch corresponded to object form by pressing the upper arrow key for yes and the lower arrow key for no. In each condition a stimulus was presented on both congruent and incongruent trials, resulting in 84 trials in the texture-verification condition (note the presentation of coloured and grey-scale textures, see Experiment 2: Design and Supplementary Materials), 80 trials in the shape verification condition, and 50\% chance level. The conditions were blocked, and the texture-verification condition was always administered first.

\section{Results}

Figure 4 reports the results. In the texture-verification condition, RDS did not differ significantly from the controls on any of the performance measures (congruent trials accuracy, incongruent trials accuracy, d' or c). In the shape-verification condition, the only performance measure in which RDS differed significantly from the controls was sensitivity (d'), which was higher in RDS than in controls (d'=4.2 vs. $3.4 \pm 0.33, t(4)=2.21, p=0.045$, $\mathrm{z}=2.42)$.

[Figure 4 somewhere here] 


\section{Discussion}

Experiment 2 results did not evidence any obvious problems in simultaneous processing of two visual stimuli in RDS. Thus, RDS's failure in the colour-split condition of experiment 1 is unlikely to be explained by simultagnosia. Experiment 2 also suggests that RDS's inability to match object's features may be colour-specific, as he was not significantly impaired on the texture-verification condition. This is consistent with reports showing different processing streams for colour and texture information (Cavina-Pratesi, Kentridge, Heywood, \& Milner, 2010). However, caution is needed when interpreting the results because our study might be underpowered to show a deficit, especially in the congruent trials of the texture-verification task.

\section{Experiment 3: Colour-name matching}

\section{Methods}

\section{Participants}

RDS and the same control participants as in experiment 1.

\section{Design}

There were 3 experimental conditions. Participants matched pre-recorded, auditorily presented colour-names to visually presented colour patches (colour-patch condition), to typically coloured objects (typical object-colour condition) and to typical colours of greyscale objects (achromatic object condition).

\section{Materials}

For the colour-patch condition, we used 50 colour patches, 10 for each of the following categories: blue, brown, green, pink and yellow. Patches were chosen on the basis of being named with at least $90 \%$ consistency by healthy native French speakers in another, 
colour-naming experiment (see Supplemental Information to Siuda-Krzywicka et al., submitted). The stimuli did not include red colours because the control, colour-naming experiment did not result in 10 colour probes that would be named with high consistency as red. In the conditions involving objects we used the same stimuli as in experiment 1.

\section{Procedure}

Each trial started with a 500-ms fixation point, followed by the simultaneous presentation of a spoken colour name and a visual stimulus (colour patch or object), located centrally on the monitor screen (see Figure 5). Participants had to judge whether the colour name matched the colour patch, the colour of the typically coloured object, or the typical colour of a grey-scale object. Participants pressed the upper arrow key of the computer keyboard for yes, and the lower arrow key for no. For each condition, the auditorily presented colour name was congruent with the visual stimulus in half of the trials (50\% chance level). Overall, participants completed 420 trials, that is 100 in the colour patch condition and 160 in the two object conditions. Each participant was presented with identical colour-name - colour combinations. The conditions were blocked. To avoid interference between object-colour and achromatic conditions, all participants performed first the colour patch condition, then the typical colour of greyscale object condition, and finally the typically coloured objects condition.

\section{Results}

When matching colour names to colour patches and typically coloured objects, RDS answered "no" more often than "yes", showing a pathologically conservative response bias $(\mathrm{c}=1.08$ vs. $-0.43 \pm 0.22$ in the control group for colour patches, $t(10)=6.6, p<0.001$; and $c$ $=1.04$ vs. $0.38 \pm 0.22$ for typically coloured objects, $\mathrm{t}(10)=2.85, \mathrm{p}=0.009, \mathrm{z}=2.98$ ). His performance was significantly more impaired in congruent trials, i.e. the trials where the visual colour matched the colour name $(42 \%$ vs. $99 \pm 1 \%$ correct in the colour-patches 
condition, $\mathrm{t}(10)=-52.17, \mathrm{p}<0.001$, effect size $\mathrm{z}=-54.48$; and $39 \%$ vs. $97 \pm 2 \%$ in the objectcolour, $\mathrm{t}(10)=-26.55, \mathrm{p}<0.001, \mathrm{z}=-27.76)$, than in incongruent trials $(98$ vs. $94 \pm 2 \%$ correct in the colour-patches condition, $\mathrm{t}(10)=1.70, \mathrm{p}=0.06, \mathrm{z}=1.77$; and $96 \%$ vs $99 \pm 2 \%$ in the typically coloured objects condition, $\mathrm{t}(10)=-1.53, \mathrm{p}=0.077, \mathrm{z}=-1.61)$. There was a significant dissociation between congruent and incongruent trails in both conditions (RSDT p $<0.001$ ).

RDS presented a different pattern of performance in matching colour names to typical colours of grey-scale objects. Despite performing well above chance level, compared with controls RDS was impaired on both congruent and incongruent trials (incongruent trials accuracy, $86 \%$ vs. $97 \pm 3 \%, \mathrm{t}(10)=-3.18, \mathrm{p}=0.005, \mathrm{z}=-3.32$; congruent trials accuracy, $76 \%$ vs. $95 \pm 5 \%, \mathrm{t}(10)=-3.93, \mathrm{p}=0.002, \mathrm{z}=-4.10)$. The difference between RDS's accuracy between congruent and incongruent trials was not significantly greater than in controls (RSDT $\mathrm{p}=0.360)$, neither was his response bias ( 0.19 vs. $0.11 \pm 0.18, \mathrm{p}=0.349)$. Importantly, on congruent trials RDS performed significantly better when matching colour names to greyscale objects than to typically coloured objects ( 76 vs. $39 \%$ correct, RSDT p $<0.001$ ).

[Figure 5 somewhere here]

\section{Discussion}

RDS performed better in the achromatic than in the two chromatic conditions, in particular since he did not show a conservative bias. This suggests that RDS could use his verbal colour knowledge to name the memory colour of grey-scale objects, but could not name the visually presented colours. It is particularly interesting that he did not recur to verbal colour knowledge in the object-colour condition, where the name of memory colour could be retrieved from both, visual and semantic information. 
It might be that in the object-colour condition the task instructions drove RDS's attention away from the object's shape and focused it on the colour. However, attention is not likely to divide between two features when those features are part of a single object (Blaser, Pylyshyn, \& Holcombe, 2000). It is thus unlikely that attending to object colour would compete with attending to object shape. A more plausible scenario is that RDS's default strategy was to encode the coloured object as a single entity, rather than in separate channels of colour and shape. After the encoding phase, RDS attempted to match the heard colour name to the visual stimulus, but failed, just like he failed in the colour-patch condition. This finding suggest that RDS was more likely to encode typically coloured objects as coloured templates, joint representations of colour and shape, rather than associations between separate representations of shapes and colours. This would support the Surface+Shape model of visual cognition (Tanaka et al., 2001).

\section{General discussion}

We tested rival models of the organisation of visual colour knowledge in a stroke patient, RDS, who showed impaired naming of visually presented colours with spared object identification and verbal colour knowledge (e.g., the ability to state that bananas are typically yellow). RDS was able to distinguish between typically and atypically coloured objects, presenting intact visual colour knowledge. However, he could not match colour names to colour patches and to typically coloured objects; neither could he match a colour patch to a memory colour of a grey-scale object. To the best of our knowledge, this is the first case of functional segregation between spared processing of coloured objects and impaired linking of object's shapes and colours. Our results bring insights into the organization of visual colour knowledge, and its relationship with language. 


\section{The cognitive organization of visual colour knowledge}

RDS's pattern of performance supports the organization of visual colour knowledge proposed in the Shape+Surface model of visual recognition (Tanaka et al., 2001). According this model, visual colour knowledge is stored as a set of coloured templates, rather than in a system of associations between separate representations of achromatic shapes and abstract colours. Our results are consistent with psychophysical and fMRI findings. The information about colour is automatically retrieved when observers view achromatic object shapes (Bannert \& Bartels, 2013; Hansen, Olkkonen, Walter, \& Gegenfurtner, 2006; Vandenbroucke, Fahrenfort, Meuwese, Scholte, \& Lamme, 2014; Witzel \& Hansen, 2015). Grey-scale versions of colour diagnostic objects are recognized slower than their coloured equivalents (review in Bramão et al., 2011); and colours of atypically coloured objects (e.g. a purple banana) are harder to identify than colours of typically coloured objects (e.g. yellow banana, Naor-Raz et al., 2003). The visual representation of colour-diagnostic objects may thus form a Gestalt made of joint representations of shape and colour. Considering that object recognition is driven predominantly by shape, in such Gestalt the weight of shape and colour features are not equal, with shape being more functionally diagnostic than colour.

\section{The relationship between colour knowledge and colour naming}

RDS's impaired performance in linking colour patches to grey-scale objects, and in matching colour names to colour patches and typically coloured objects shared striking similarities. In the three cases, RDS was impaired on congruent trials, i.e. when a colour name matched a visual stimulus; and presented a pathological, conservative bias (Figure 6). Could breaking down the shape+colour Gestalts into separate features of colour and shape involve colour language? 
[Figure 6 somewhere here]

Studies in cognitive development and cultural anthropology imply that processing colourdiagnostic objects in separate channels of colour and shape might not be the default way in which humans conceptualize their environment. Studies on cognitive development showed that young children struggle when asked to sort or match objects according to their colours (Kowalski \& Zimiles, 2006; Sandhofer \& Smith, 1999; Smith, 1984), as if colour was not easily accessible to judge object similarity. A child starts to understand that colour can be independent from particular objects at the same time when he or she acquires their first colour names (Baldwin, 1989; Kowalski \& Zimiles, 2006; O’Hanlon \& Roberson, 2006; Soja, 1994). Children might not attend to colours as interesting object properties unless their attention is explicitly drawn to it with the use of colour names, because colours, contrary to shape, are relatively uninformative about object's function (Baldwin, 1989; O'Hanlon \& Roberson, 2006; Sandhofer \& Smith, 1999). Colours might also be especially difficult to conceptualize. The same colours can characterize objects from completely different object classes, such as apples and fire engines. Finding common traits between these objects, such as a similar colour, requires relational thinking about them while disregarding the vast differences in their shape and function. Explicit verbal cues, such as colour names, may facilitate this process (Kowalski \& Zimiles, 2006; see also Pitchford \& Mullen, 2001 for the discussion of color as a special case of conceptualization).

Similar suggestions were made based on research in linguistic anthropology. In some remote cultures inhabitants rarely use colours to identify objects or differentiate between objects (Kuschel \& Monberg, 1974; Levinson, 2000). Using colour names and detaching colour from object shape seem to be related. Levinson (2000) suggested that "it is only when colour is detachable from objects with inherent colours that a colour terminology has any real 
communicational function" (p. 41), and pointed to the discovery of paints and dyes as a possible reason underlying the development of colour names. Consistent with this hypothesis, Gibson and colleagues (2017) showed that Tsimane, an indigenous Amazonian tribe, were more prone to use colour names when identifying non colour-diagnostic, artificial objects, such as cups or ropes, rather than natural objects (e.g. bananas or leaves, see their figure 6).

The conceptualization of colour as an abstract property, detachable from object, may depend on the use of colour names. Our results, together with the reports on aphasic patients (Basso et al., 1985; De Renzi \& Spinnler, 1967), provide evidence for this account on a neurobiological level. RDS, whose lesion prevented him from retrieving names of visually presented colours, was also impaired in linking colours and shapes of colour diagnostic objects. Consistent with this association, some aphasic patients have difficulties in picking up the correct crayon to colour line drawings of objects (Basso et al., 1985, 1976; Cohen \& Kelter, 1979; De Renzi \& Spinnler, 1967; Varney, 1982).

There are multiple ways in which language can interact with perception and thought (reviewed in Wolff \& Holmes, 2011). Concerning the relation between object colour and object shape, language could act as an augmenter (Christie \& Gentner, 2012; Wolff \& Holmes, 2011). In this view, language (colour names) could introduce the idea of colour as a separate, object-independent entity, thus "augmenting" thought by offering a new equivalence class to organise knowledge about the environment. Evidence from young children and remote cultures suggest that such equivalence class is not available unless developed by the acquisition of colour names. However, such an equivalence class could in principle become internalised and language-independent in development (e.g. Loewenstein \& Gentner, 2005). Observations that neurological patients with language impairments struggle in relating abstract colour to object shapes suggest otherwise, showing that language is operative in detaching colour from object representation even in the adult cognitive system. 


\section{The neuro-functional organization of visual colour knowledge}

It was recently suggested that cortical colour processing in the ventral occipitotemporal cortex is organised in a hierarchy (Conway, 2018), similar to the idea of the hierarchical processing of faces (Freiwald \& Tsao, 2010). In such a hierarchy, posterior colour-biased regions encode single hues; the central colour-biased regions group the hues into behaviourally relevant ensembles, i.e. colour categories; and the anterior colour-regions represent the memory of colours typical for given objects, i.e. object-colour knowledge (Conway, 2018).

Our results further extend this analogy (see Figure 7). The content of object-colour knowledge - coloured templates, or joint representations of shape and colour - could constitute a visual Gestalt, similar to the idea of holistic processing of faces (for review see Maurer, Grand, \& Mondloch, 2002). The coloured templates could be processed in the anterior colour region, selective to both shape and colour (Lafer-Sousa, Conway, \& Kanwisher, 2016) and built upon inputs from the shape-selective patches in the lateral parts of the inferior temporal cortex (Grill-Spector \& Weiner, 2014), and the central colour regions, located in its more mesial portions (Lafer-Sousa et al., 2016; Rosenthal et al., 2018; Zeki \& Marini, 1998). In this view, colour agnosia could be understood as a deficit in object-colour knowledge (Miceli et al., 2001; Stasenko, Garcea, Dombovy, \& Mahon, 2014), and would be caused by brain damage affecting the neural substrates of the colour and shape Gestalts.

We also speculate that the cultural invention of paints and dyes that might have led to the formation of colour as an abstract (object-independent) semantic category, could have also led to the neural reorganization of cortical circuits that process colours, similar to the reorganization of object-selective structures with the development of writing systems (Dehaene \& Cohen, 2007). As a result, the left colour-selective regions, thanks to their proximity to language regions, could support the ability to detach colours from objects, and to 
link these abstract colour concepts to their names. Colour naming deficits typically occur after damage to the left mesial occipito-temporal cortex, including the lingual gyrus and the parahippocampal areas (Damasio \& Damasio, 1983). Lesions causing colour-naming deficits frequently overlap with the described locations of the ventral colour-selective regions (LaferSousa et al., 2016). The left colour-selective regions could therefore connect visually presented colours with colour names (Siuda-Krzywicka et al., submitted) .

[Figure 7 somewhere here]

Does the ability to abstract colours from objects rely on the same cortical circuits as colour naming? RDS's lesion is well-delimited but quite extensive. This leaves open the possibility that the cortical maps of conceptual colour processing and of colour naming are distinct but closely neighbouring. Previous reports on impaired colour-object matching in aphasic patients did not include the lesion locations, thus it is unclear what are the exact lesion sites that cause impairments in colour conceptualization (Basso et al., 1985, 1976; Cohen \& Kelter, 1979; De Renzi \& Spinnler, 1967). However, Varney (1982) reported that in his study all of the aphasic patients showing deficits on the colour-object matching task were also alexic, which suggests that colour abstraction is computed within the left ventral visual stream. The fact that colour naming and colour conceptualization may be dissociated comes from a seminal case report of visuo-verbal disconnection specific to colours (Geschwind \& Fusillo, 1966). The patient reported by Geschwind and Fusillo (1966) was able to pick a typical colour for grey-scale objects, while not being able to name visually presented colours. The exact relationship between abstracting colour from objects and colour naming remains to be established.

\section{Conclusions}

Across the three experiments, patients RDS presented an association between colourselective naming deficits and impaired matching of colour patches to visually presented object 
shapes. Thus, in RDS the bottom-up visual colour information was disconnected from the topdown colour language and colour knowledge. Based on RDS's performance patterns and lesion location, and further examples from the literature, we suggest that (1) visual colour knowledge is organized as a set of coloured templates rather than associations between separate representations of achromatic shapes and colours; (2) language is involved in abstracting colours from their objects; and (3) the neural hierarchy of colour processing in occipito-temporal cortex is lateralized, with left-hemisphere colour patches engaged in abstracting colours from objects and linking them to colour names.

\section{Acknowledgments}

We are deeply grateful to Mr. RDS for his patience and good humour during endless hours of testing. We also thank Dr Karynne Moreau, Noelia Cools and Sophie Ferrieux for patient referral and clinical follow-up, and Michel Thiebaut de Schotten for advice and discussion. The research leading to these results was promoted by the Inserm (protocol C13-41), was approved by the Ethical Committee Ile-de-France I, and has received funding from the program "Investissements d'avenir" ANR-10- IAIHU-06. K.S.K. was funded by the École des Neurosciences Paris Île de France. C.W. was supported by the ERC Advanced Grant FEEL No. 323674 and the 'Cardinal Mechanisms of Perception' grant No SFB TRR 135 from the Deutsche Forschungsgemeinschaft.

\section{Declaration of interest statement}

Authors declare no conflict of interest. 


\section{References}

Baldwin, D. A. (1989). Priorities in children's expectations about object label reference: form over color. Child Development, 60(6), 1291-1306. http://doi.org/10.1111/j.14678624.1989.tb04003.x

Bannert, M. M., \& Bartels, A. (2013). Decoding the yellow of a gray banana. Current Biology : CB, 23(22), 2268-72. http://doi.org/10.1016/j.cub.2013.09.016

Bartleson, C. J. (1960). Memory colors of familiar objects. J. Opt. Soc. Am., 50(1), 73-77. http://doi.org/10.1364/JOSA.50.000073

Bartolomeo, P., Bachoud-Lévi, A. C., \& Denes, G. (1997). Preserved imagery for colours in a patient with cerebral achromatopsia. Cortex; a Journal Devoted to the Study of the Nervous System and Behavior, 33(2), 369-78. http://doi.org/10.1016/S00109452(08)70012-1

Basso, A., Capitani, E., Luzzatti, C., Spinnler, H., \& Zanobio, M. E. (1985). Different basic components in the performance of Broca's and Wernicke's aphasics on the colour-figure matching test. Neuropsychologia, 23(1), 51-59. http://doi.org/10.1016/00283932(85)90043-0

Basso, A., Faglioni, P., \& Spinnler, H. (1976). Non-verbal colour impairment of aphasics. Neuropsychologia, 14(2), 183-193. http://doi.org/10.1016/0028-3932(76)90047-6

Biederman, I., \& Ju, G. (1988). Surface versus edge-based determinants of visual recognition. Cognitive Psychology, 20(1), 38-64. http://doi.org/10.1016/0010-0285(88)90024-2

Blaser, E., Pylyshyn, Z. W., \& Holcombe, A. O. (2000). Tracking an object through feature space. Nature, 408(6809), 196-199. http://doi.org/10.1038/35041567

Bourlon, C., Chokron, S., Bachoud-Lévi, A. C., Coubard, O., Bergeras, I., Moulignier, A., ... Bartolomeo, P. (2009). Normalisation d'une batterie d'évaluation de l'imagerie mentale visuelle et de la perception visuelle. Revue Neurologique, 165(12), 1045-1054. 
http://doi.org/10.1016/j.neurol.2009.04.010

Bramão, I., Reis, A., Petersson, K. M., \& Faísca, L. (2011). The role of color information on object recognition: a review and meta-analysis. Acta Psychologica, 138(1), 244-53. http://doi.org/10.1016/j.actpsy.2011.06.010

Cavina-Pratesi, C., Kentridge, R. W., Heywood, C. A., \& Milner, A. D. (2010). Separate Channels for Processing Form, Texture, and Color: Evidence from fMRI Adaptation and Visual Object Agnosia. Cerebral Cortex, 20, 2319-2332. http://doi.org/10.1093/cercor/bhp298

Christie, S., \& Gentner, D. (2012). Language and Cognition in Development. The Cambridge Handbook of Psycholinguistics, 653-673. http://doi.org/10.1017/cbo9781139029377.044

Cohen, R., \& Kelter, S. (1979). Cognitive Impairment of Aphasics in a Colour-To-Picture Matching Task. Cortex, 15(2), 235-245. http://doi.org/10.1016/S0010-9452(79)80028-3

Conway, B. R. (2018). The Organization and Operation of Inferior Temporal Cortex. Annu. Rev. Vis. Sci. http://doi.org/10.1146/annurev-vision-091517-034202

Conway, B. R., Chatterjee, S., Field, G. D., Horwitz, G. D., Johnson, E. N., Koida, K., \& Mancuso, K. (2010). Advances in color science: from retina to behavior. The Journal of Neuroscience : The Official Journal of the Society for Neuroscience, 30(45), 14955-63. http://doi.org/10.1523/JNEUROSCI.4348-10.2010

Crawford, J. R., \& Garthwaite, P. H. (2005). Testing for suspected impairments and dissociations in single-case studies in neuropsychology: evaluation of alternatives using Monte Carlo simulations and revised tests for dissociations. Neuropsychology, 19(3), 318-31. http://doi.org/10.1037/0894-4105.19.3.318

Crawford, J. R., \& Howell, D. C. (1998). Comparing an Individual's Test Score Against Norms Derived from Small Samples. The Clinical Neuropsychologist (Neuropsychology, Development and Cognition: Section D), 12(4), 482-486. 
http://doi.org/10.1076/clin.12.4.482.7241

Damasio, A., \& Damasio, H. (1983). The anatomic basis of pure alexia. Neurology, 33(12), 1573-83. http://doi.org/10.1212/WNL.33.12.1573

Davidoff, J. (2001). Language and perceptual categorisation. Trends in Cognitive Sciences, 5(9), 382-387.

Davidoff, J., \& Mitchell, P. (1993). The colour cognition of children. Cognition, 48(2), 121137. http://doi.org/10.1016/0010-0277(93)90027-S

De Renzi, E., \& Spinnler, H. (1967). Impaired Performance on Color Tasks in Patients with Hemispheric Damage. Cortex, 3(2), 194-217. http://doi.org/10.1016/S00109452(67)80012-1

De Vreese, L. P. (1991). Two systems for colour-naming defects: Verbal disconnection vs colour imagery disorder. Neuropsychologia, 29(1), 1-18. http://doi.org/10.1016/00283932(91)90090-U

Dehaene, S., \& Cohen, L. (2007). Cultural Recycling of Cortical Maps. Neuron, 56(2), 384398. http://doi.org/10.1016/J.NEURON.2007.10.004

Freiwald, W. A., \& Tsao, D. Y. (2010). Functional Compartmentalization and Viewpoint Generalization Within the Macaque Face-Processing System. Science, 330(6005), 845851. http://doi.org/10.1126/science. 1194908

Gegenfurtner, K. R., \& Kiper, D. C. (2003). Color vision. Annual Review of Neuroscience, 26(1), 181-206. http://doi.org/10.1146/annurev.neuro.26.041002.131116

Geschwind, N., \& Fusillo, M. (1966). Color-naming defects in association with alexia. Archives of Neurology, 15, 137-146. http://doi.org/10.1001/archneur.1966.00470140027004

Gibson, E., Futrell, R., Jara-Ettinger, J., Mahowald, K., Bergen, L., Ratnasingam, S., ... Conway, B. R. (2017). Color naming across languages reflects color use. Proceedings of 
the National Academy of Sciences of the United States of America, 114(40), 1078510790. http://doi.org/10.1073/pnas.1619666114

Gleason, T. R., Fiske, K. E., \& Chan, R. K. (2004). The verbal nature of representations of the canonical colors of objects. Cognitive Development, 19(1), 1-14. http://doi.org/10.1016/S0885-2014(03)00044-3

Grill-Spector, K., \& Weiner, K. S. (2014). The functional architecture of the ventral temporal cortex and its role in categorization. Nature Reviews. Neuroscience, 15(8), 536-48. http://doi.org/10.1038/nrn3747

Hansen, T., Olkkonen, M., Walter, S., \& Gegenfurtner, K. R. (2006). Memory modulates color appearance. Nature Neuroscience, 9(11), 1367-8. http://doi.org/10.1038/nn1794

Ishihara, S. (1974). Tests for colour-blindness. Tokio: Kanehara Shup.

Kowalski, K., \& Zimiles, H. (2006). The relation between children's conceptual functioning with color and color term acquisition. Journal of Experimental Child Psychology, 94(4), 301-321. http://doi.org/10.1016/j.jecp.2005.12.001

Kuschel, R., \& Monberg, T. (1974). "We Don't Talk Much About Colour Here”: A Study of Colour Semantics on Bellona Island. Man, New Series, 9(2), 213-242.

Lafer-Sousa, R., Conway, B. R., \& Kanwisher, N. G. (2016). Color-Biased Regions of the Ventral Visual Pathway Lie between Face- and Place-Selective Regions in Humans, as in Macaques. Journal of Neuroscience, 36(5), 1682-1697. http://doi.org/10.1523/JNEUROSCI.3164-15.2016

Levinson, S. C. (2000). Yeli Dnye and the Theory of Basic Color Terms. Journal of Linguistic Anthropology, 10(1), 3-55. http://doi.org/10.1525/jlin.2000.10.1.3

Loewenstein, J., \& Gentner, D. (2005). Relational language and the development of relational mapping. Cognitive Psychology, 50(4), 315-353. http://doi.org/10.1016/j.cogpsych.2004.09.004 
Mathôt, S., Schreij, D., \& Theeuwes, J. (2012). OpenSesame: An open-source, graphical experiment builder for the social sciences. Behavior Research Methods, 44(2), 314-324. http://doi.org/doi:10.3758/s13428-011-0168-7

Maurer, D., Grand, R. Le, \& Mondloch, C. J. (2002). The many faces of configural processing. Trends in Cognitive Sciences, 6(6), 255-260. http://doi.org/10.1016/S13646613(02)01903-4

Miceli, G., Fouch, E., Capasso, R., Shelton, J. R., Tomaiuolo, F., \& Caramazza, A. (2001). The dissociation of color from form and function knowledge. Nature Neuroscience, 4(6), 662-667. http://doi.org/10.1038/88497

Naor-Raz, G., Tarr, M. J., \& Kersten, D. (2003). Is color an intrinsic property of object representation? Perception, 32(6), 667-680. http://doi.org/10.1068/p5050

O’Hanlon, C. G., \& Roberson, D. (2006). Learning in context: Linguistic and attentional constraints on children's color term learning. Journal of Experimental Child Psychology, 94(4), 275-300. http://doi.org/10.1016/J.JECP.2005.11.007

O’Neill-Biba, M., Sivaprasad, S., Rodriguez-Carmona, M., Wolf, J. E., \& Barbur, J. L. (2010). Loss of chromatic sensitivity in AMD and diabetes: a comparative study. Ophthalmic and Physiological Optics, 30(5), 705-716. http://doi.org/10.1111/j.14751313.2010.00775.x

Oldfield, R. C. (1971). The assessment and analysis of handedness: The Edinburgh inventory. Neuropsychologia. http://doi.org/10.1016/0028-3932(71)90067-4

Pitchford, N. J., \& Mullen, K. T. (2001). Conceptualization of Perceptual Attributes: A Special Case for Color? Journal of Experimental Child Psychology, 80, 289-314. http://doi.org/10.1006/jecp.2001.2634

Regier, T., \& Kay, P. (2009). Language, thought, and color: Whorf was half right. Trends in Cognitive Sciences, 13(10), 439-446. http://doi.org/10.1016/j.tics.2009.07.001 
Rodrigues, M. D. A., Adda, C. C., Cristina, M., Lucia, D. S., Scaff, M., \& Miotto, E. C. (2008). Cognitive deficits associated with optic aphasia Neuropsychological contribution to a differential diagnosis, 2(2), 151-154.

Rosenthal, I., Ratnasingam, S., Haile, T., Eastman, S., Fuller-Deets, J., \& Conway, B. R. (2018). Color statistics of objects, and color tuning of object cortex in macaque monkey. Journal of Vision, 18(11), 1. http://doi.org/10.1167/18.11.1

Rossion, B., \& Pourtois, G. (2004). Revisiting Snodgrass and Vanderwart's object pictorial set: The role of surface detail in basic-level object recognition. Perception, 33(2), 217236. http://doi.org/10.1068/p5117

Sandhofer, C. M., \& Smith, L. B. (1999). Learning color words involved learning a system of mappings. Developmental Psychology, 35(3), 668-679. Retrieved from http://psycnet.apa.org/fulltext/1999-05027-006.html

Siuda-Krzywicka, K., Boros, M., Bartolomeo, P., \& Witzel, C. (2019). The biological bases of colour categories: from goldfish to the human brain. Cortex. Retrieved from https://doi.org/10.1016/j.cortex.2019.04.010

Siuda-Krzywicka, K., Witzel, C., Chabani, E., Seidel Malkinson, T., Taga, M., Coste, C., ... Bartolomeo, P. (n.d.). Colour categorisation independent of colour naming. Under Review.

Smith, L. B. (1984). Young Children's Understanding of Attributes and Dimensions: A Comparison of Conceptual and Linguistic Measures. Child Development, 55(2), 363. http://doi.org/10.2307/1129949

Snodgrass, J. G., \& Vanderwart, M. (1980). A standardized set of 260 pictures: norms for name agreement, image agreement, familiarity, and visual complexity. Journal of Experimental Psychology. Human Learning and Memory, 6(2), 174-215. Retrieved from http://www.ncbi.nlm.nih.gov/pubmed/7373248 
Soja, N. N. (1994). Young Children's Concept of Color and Its Relation to the Acquisition of Color Words. Child Development, 65(3), 918. http://doi.org/10.2307/1131428

Stanislaw, H., \& Todorov, N. (1999). Calculation of signal detection theory measures. Behavior Research Methods, Instruments, \{\&\} Computers, 31(1), 137-149. http://doi.org/10.3758/BF03207704

Stasenko, A., Garcea, F. E., Dombovy, M., \& Mahon, B. Z. (2014). When concepts lose their color: A case of object-color knowledge impairment. Cortex, 58, 217-238. http://doi.org/10.1016/j.cortex.2014.05.013

Tanaka, J., Weiskopf, D., \& Williams, P. (2001). The role of color in high-level vision. Trends in Cognitive Sciences, 5(5), 211-215. http://doi.org/10.1016/S13646613(00)01626-0

Vandenbroucke, a R. E., Fahrenfort, J. J., Meuwese, J. D. I., Scholte, H. S., \& Lamme, V. a F. (2014). Prior Knowledge about Objects Determines Neural Color Representation in Human Visual Cortex. Cerebral Cortex (New York, N.Y. : 1991). http://doi.org/10.1093/cercor/bhu224

Varney, N. R. (1982). Colour association and "colour amnesia" in aphasia. Journal of Neurology Neurosurgery and Psychiatry, 45(3), 248-252. http://doi.org/10.1136/jnnp.45.3.248

Witzel, C. (2018). Misconceptions About Colour Categories. Review of Philosophy and Psychology, 1-42. http://doi.org/10.1007/s13164-018-0404-5

Witzel, C., \& Gegenfurtner, K. (2014). Memory Color. In Encyclopedia of Color Science and Technology. Springer Science+Business Media. http://doi.org/10.1007/978-3-64227851-8_58-8

Witzel, C., \& Gegenfurtner, K. R. (2018). Color Perception: Objects, Constancy, and Categories. Annual Review of Vision Science, 4(1), 16.1-16.25. 
http://doi.org/10.1146/annurev-vision-091517-034231

Witzel, C., \& Hansen, T. (2015). Memory effects on color perception. In A. J. Elliot, M. D. Fairchild, \& A. Franklin (Eds.), Handbook of Color Psychology (pp. 641-659). Cambridge: Cambridge University Press. http://doi.org/10.1017/CBO9781107337930.032

Witzel, C., Valkova, H., Hansen, T., \& Gegenfurtner, K. R. (2011). Object knowledge modulates colour appearance. I-Perception, 2(1), 13-49. http://doi.org/10.1068/i0396

Wolff, P., \& Holmes, K. J. (2011). Linguistic relativity. Wiley Interdisciplinary Reviews: Cognitive Science, 2(3), 253-265. http://doi.org/10.1002/wcs.104

Zeki, S., \& Marini, L. (1998). Three cortical stages of colour processing in the human brain. Brain, 121(9), 1669-1685. http://doi.org/10.1093/brain/121.9.1669 
Figures

Figure 1

\section{Visual Colour Knowledge}
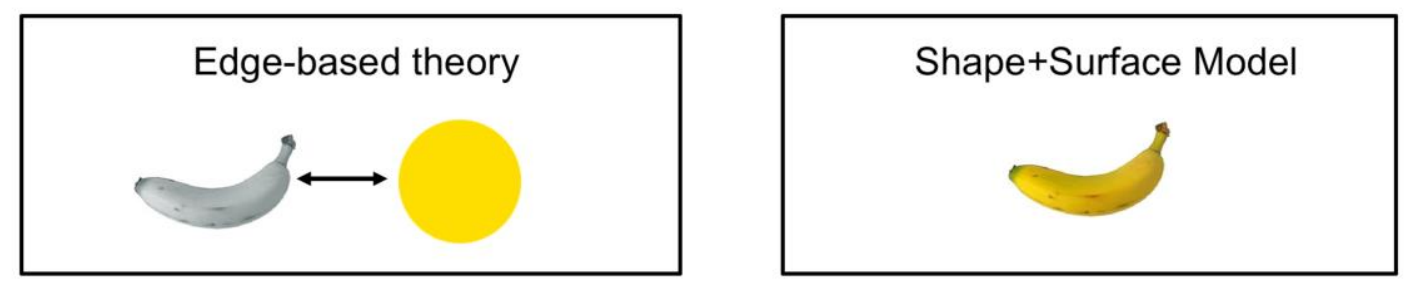

\section{Figure 2}

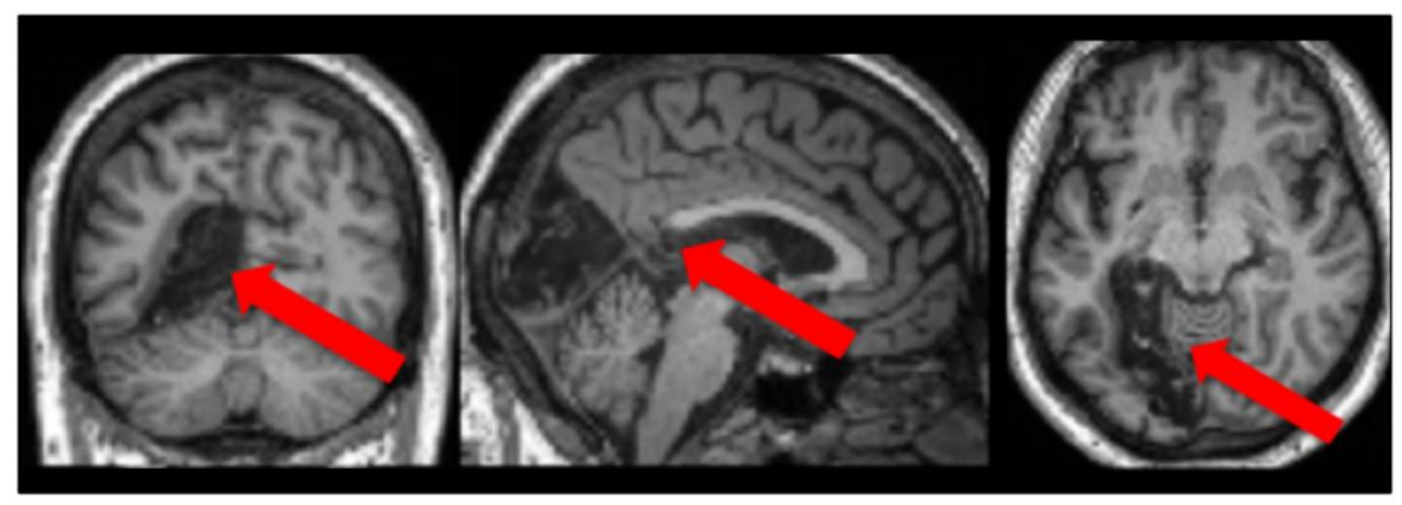


Figure 3
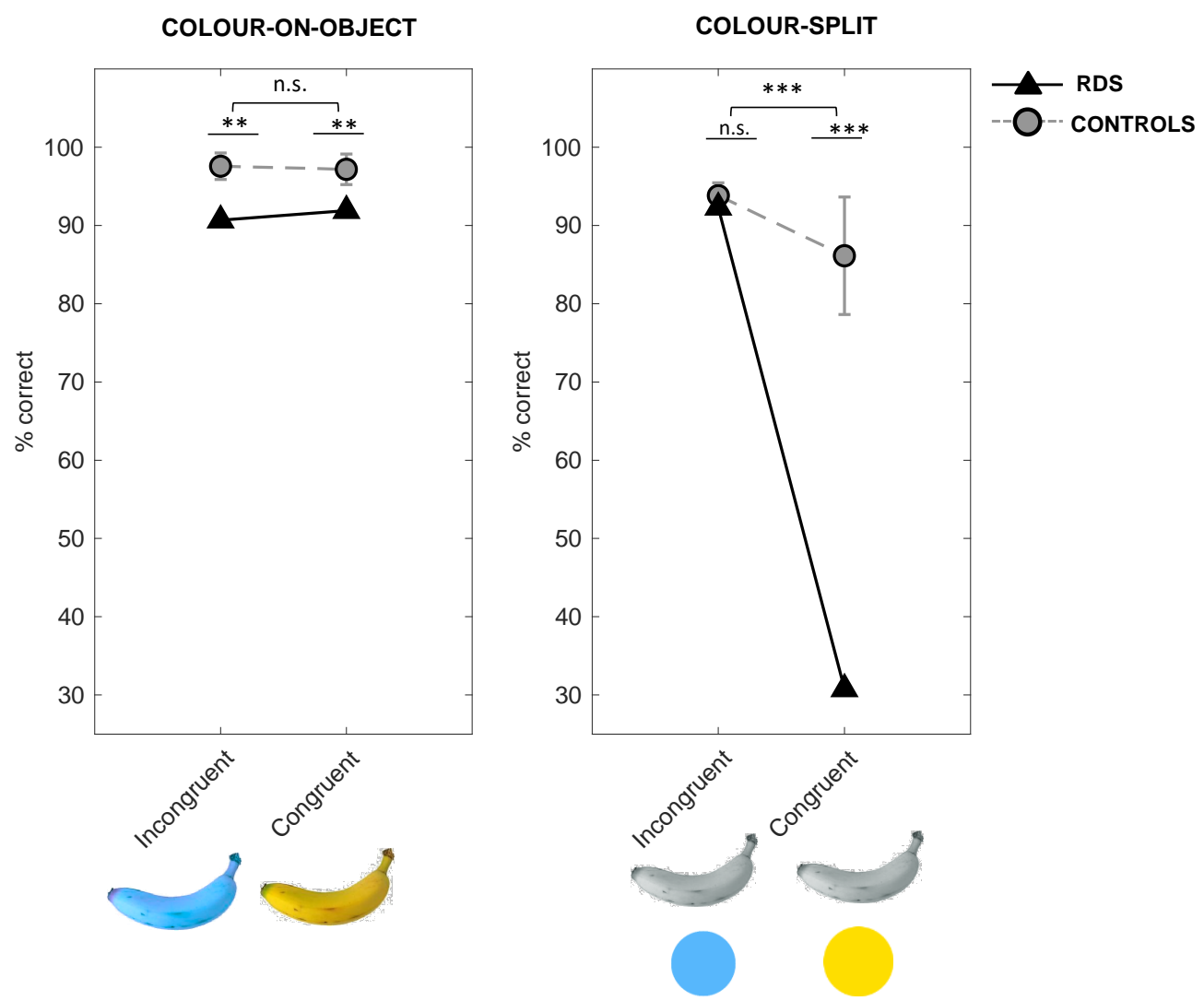
Figure 4
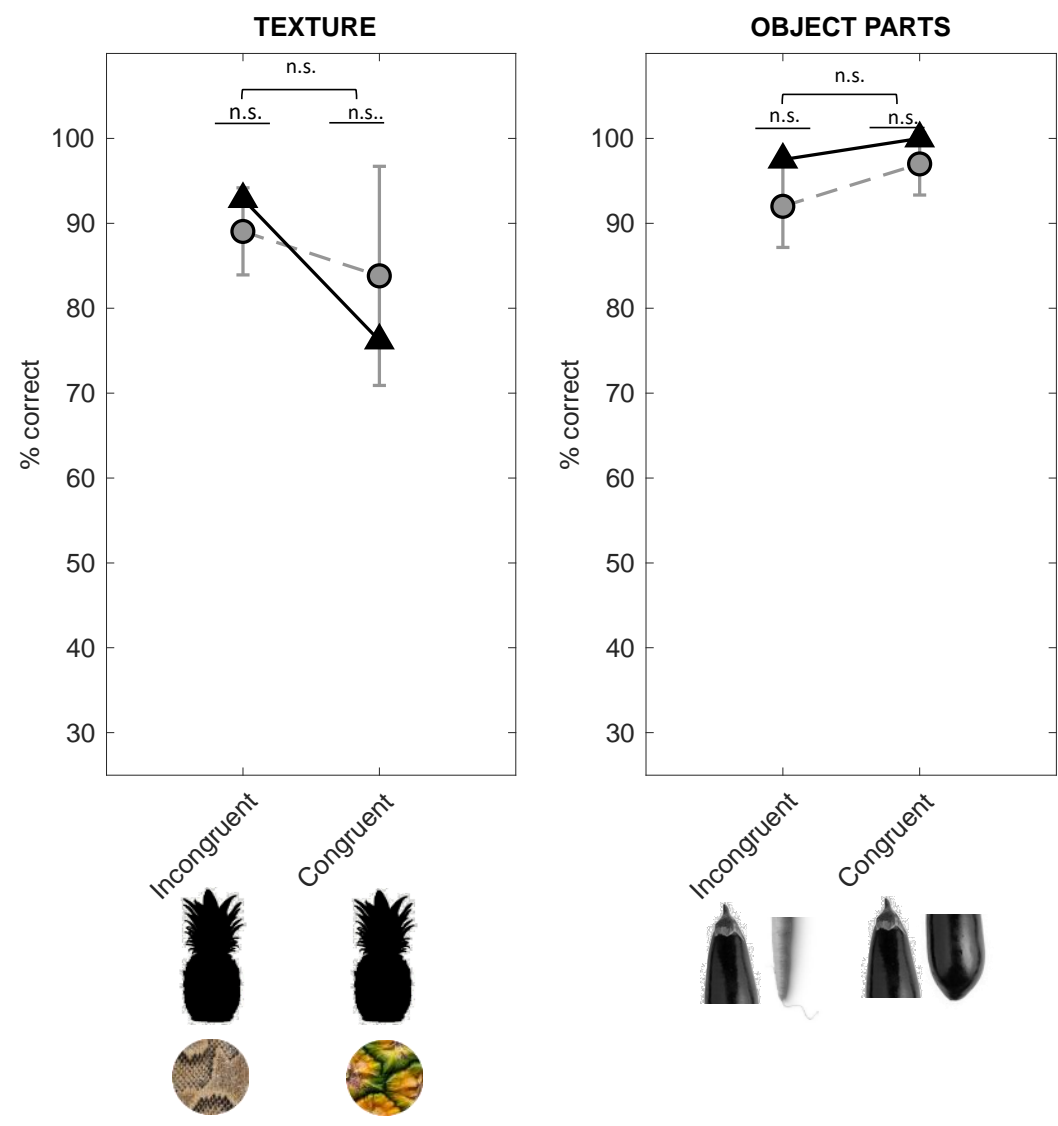
Figure 5

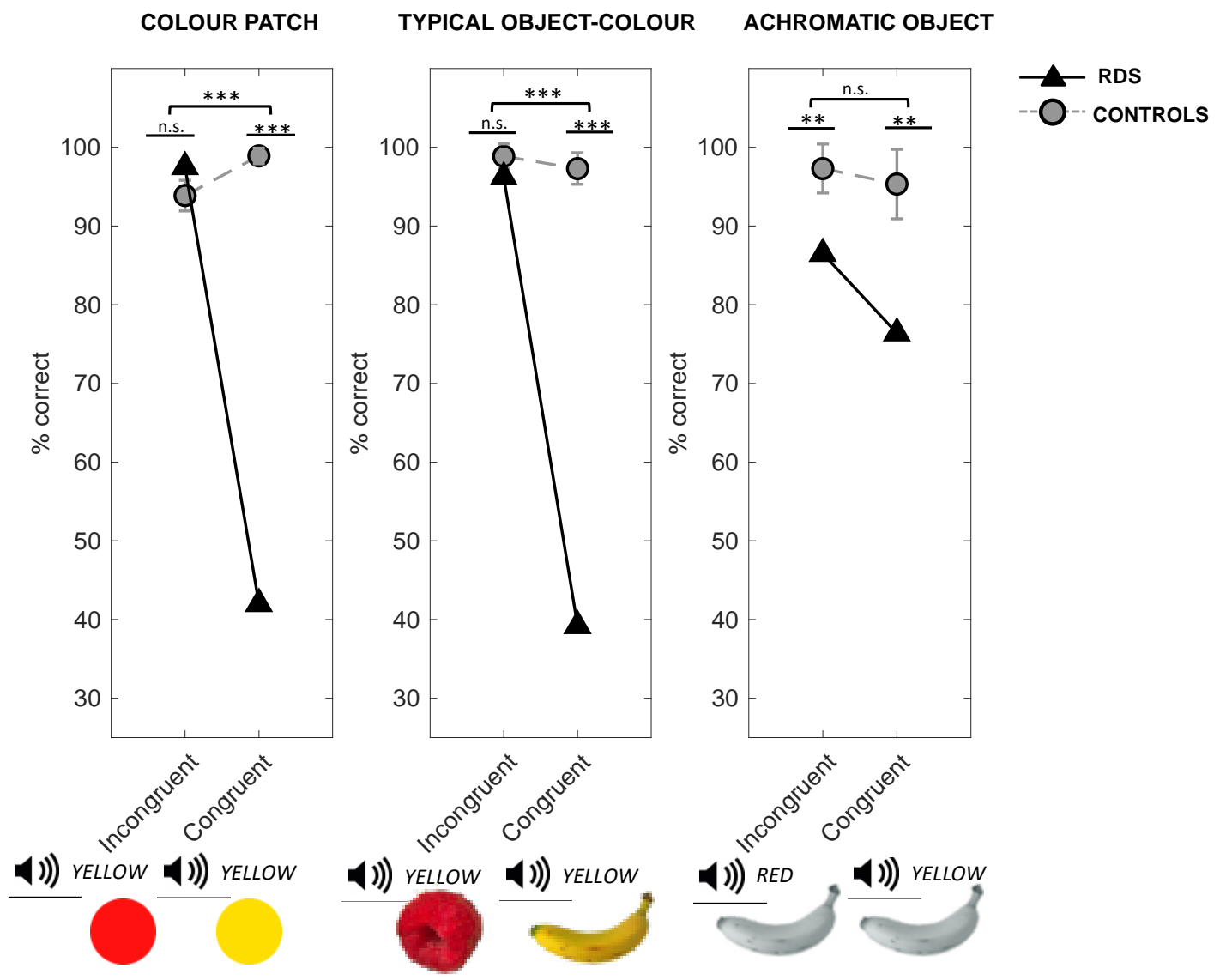


Figure 6

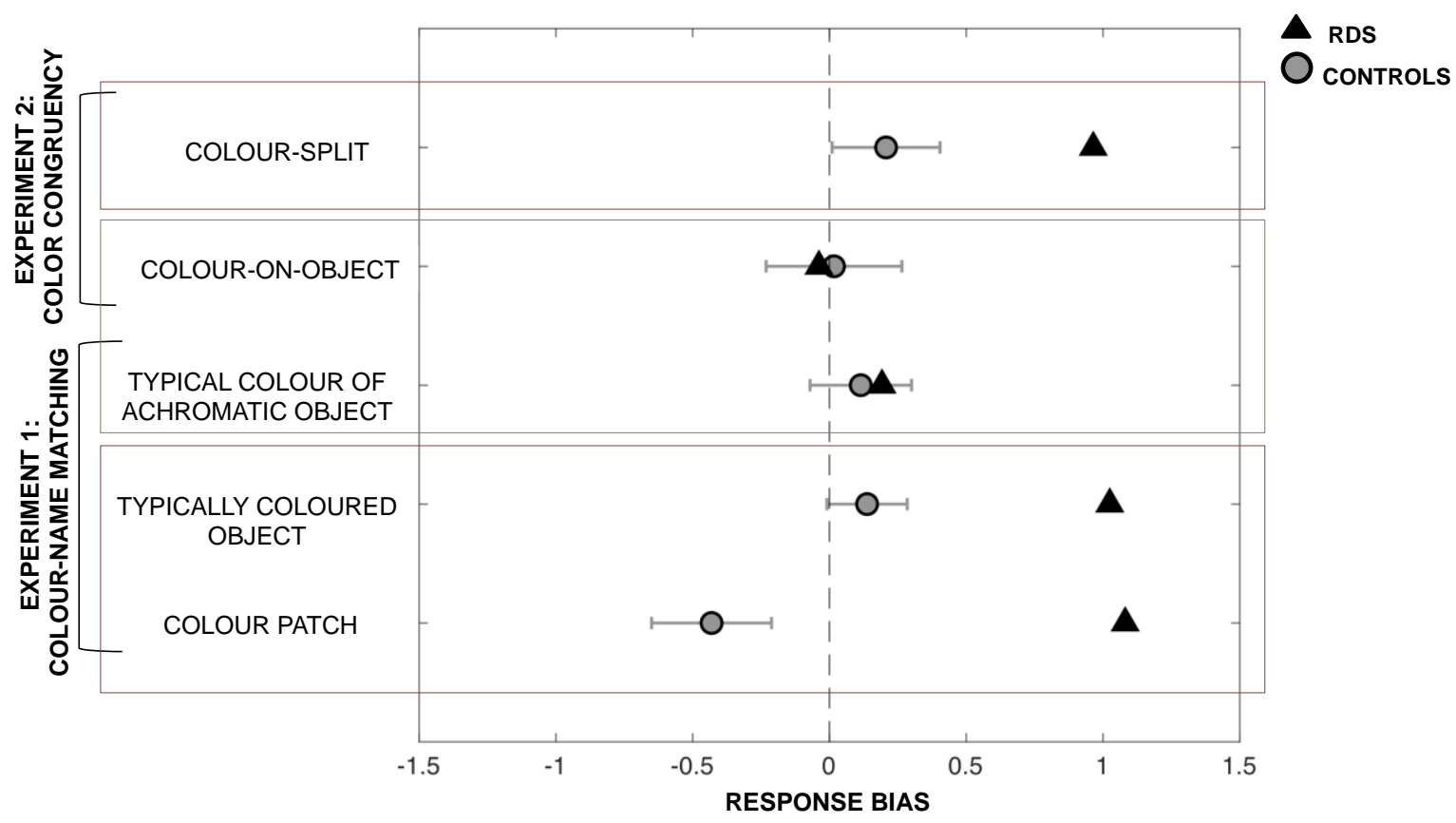


38

Figure 7

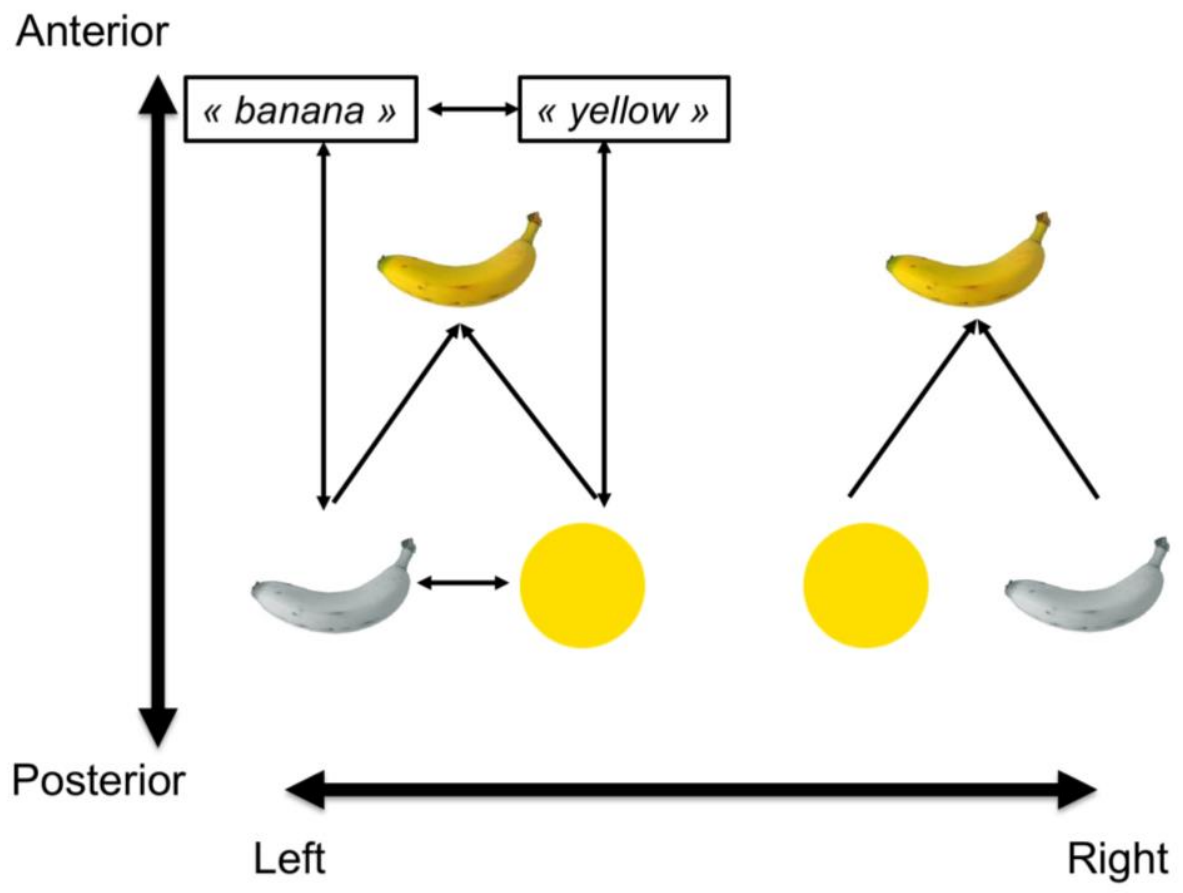




\section{Figure Legends}

Figure 1. Models of the organisation of visual colour knowledge. Edge-based theory states that knowledge about typical object colours is stored as associations between separate representations of object shapes and colours. Alternative, Shape+Surface model proposes that visual colour knowledge consists of sets of coloured templates, joint representations of colour and shape.

Figure 2. T1-weighted images of RDS's brain. Arrows indicate the lesion.

Figure 3. Experiment 1: Colour Congruency Judging. RDS's (black triangle) relatively good performance in the colour-on-object condition contrasted with impairment in the colour-split condition, where he showed strong conservative response bias. Error bars depict \pm 1 standard deviation in the control group $(n=11)$. Images below the $x$ axis show examples of trials for each condition. $* \mathrm{p}<0.05, * * \mathrm{p}<0.01, * * * \mathrm{p}<0.001$

Figure 4. Experiment 2: Property Verification Task. RDS's was not impaired in colour unrelated tasks requiring simultaneous processing of two stimuli. Error bars depict \pm 1 standard deviation in the control group $(n=5)$. Images below the $\mathrm{x}$ axis show examples of trials for each condition. $* \mathrm{p}<0.05, * * \mathrm{p}<0.01, * * * \mathrm{p}<0.001$.

Figure 5. Experiment 3: Colour-Name Matching. RDS could not match colour names to colour patches and typically coloured objects and showed a pathologically conservative response bias. At the same time, his performance pattern was different and overall better when matching colour names to colours retrieved from verbal colour knowledge. Error bars depict \pm 1 standard deviation in the control group $(n=11)$. Images below the $\mathrm{x}$ axis show examples of trials for each condition. ${ }^{*} \mathrm{p}<0.05, * * \mathrm{p}<0.01, * * * \mathrm{p}<0.001$.

Figure 6. Response bias in experiments 1 and 3. The red rectangle contains the tasks where RDS (black triangles) presented a significantly more conservative bias that the healthy controls (grey dots). The green rectangle contains the tasks where RDS's bias did not differ significantly from controls'. Error bars depict \pm 1 standard deviation in the control group.

Figure 7. A proposed model of the neuro-functional organization of object-colour knowledge along the posterior/anterior and left/right axis of the inferior occipito-temporal cortex. Most posterior parts of the occipito-temporal cortex, not illustrated here, encode single hues and line-junctions, the basic building blocks of more complex shape and colour representations (reviewed in Conway, 2018; Conway et al., 2010; Gegenfurtner \& Kiper, 2003; Grill-Spector $\&$ Weiner, 2014). Colour and shape selective regions in the central parts of the occipitotemporal cortex compute object colours (Lafer-Sousa et al., 2016; Rosenthal et al., 2018) and object shapes (Grill-Spector \& Weiner, 2014) respectively; and send it to the most anterior, colour- and shape-selective patches (Lafer-Sousa et al., 2016) that compute the joint representation of objects in their typical colour, constituting the visual colour knowledge. The intermediate stage of this model is lateralized, with left colour and shape patches being 
preferentially connected to language regions, enabling the associations between visually presented colours and shapes, and their names. The associations between colour and shape names constitute the verbal colour knowledge. This left lateralized system could also support abstracting colours from objects and conceptually relating visually presented abstract colours to object shapes. 